\title{
FACTORES ASOCIADOS A LA CITOLOGÍA ANORMAL DEL CÉRVIX. LIGA CONTRA EL CÁNCER, LIMA-PERÚ, 2010-2014
}

\author{
Mario Ernesto Cosser Herrera ${ }^{1, a}$, Manuel Jesús Loayza Alarico ${ }^{2, b}$, Pedro Javier Navarrete Mejía 3 ,c,d
}

\begin{abstract}
RESUMEN
Objetivos. El objetivo del estudio fue identificar los factores asociados a la citología anormal del cérvix en la Liga Contra el Cáncer Lima-Perú, 2010-2014, determinando la prevalencia de la citología anormal asociada a las características socio demográficas de las pacientes. Materiales y métodos. Estudio cuantitativo, observacional, analítico, retrospectivo de corte transversal, diseño no experimental. Se estudió 206,203 citologías de cérvix como población total, el instrumento de recolección de datos fue una ficha confeccionada a partir de la historia clínica de cada paciente. Para el análisis se hizo uso de estadística descriptiva y de pruebas estadísticas analíticas. Resultados. La edad media fue de 47 años con una DS \pm 13 años, se encontró $1,80 \%$ de prevalencia de citología anormal, el mayor porcentaje de esta citología estuvo en el rango de edad de 15 a 24 años con 3,06\%, que a su vez presentó el mayor porcentaje lesión intraepitelial de bajo grado con 3,04\%, y el mayor porcentaje de lesión intraepitelial de alto grado estuvo en rango de edad de 35 a 49 años con 0,34\%. Se identificó como factores asociados, principalmente el inicio de relaciones sexuales a temprana edad ( 13 a 15 años) con un $p=0,001$, el número de parejas sexuales ( 3 a más) con un $p=0,001$ y el nivel socioeconómico, a menor nivel mayor prevalencia de citología anormal. Conclusiones. Se identificó como factores asociados a la citología anormal, a aquellos relacionados a la sexualidad y nivel socioeconómico, determinándose la prevalencia de esta citología anormal en 1,80\%.
\end{abstract}

Palabras clave: Citología anormal; Lesión intraepitelial de alto grado; Lesión intraepitelial de bajo grado (Fuente: DeCS BIREME).

\section{FACTORS ASSOCIATED WITH THE ABNORMAL CYTOLOGY OF CÉRVIX. LEAGUE AGAINST THE CANCER, LIMA-PERU, 2010-2014}

\begin{abstract}
Objectives. The objective of the study was to identify factors associated with abnormal cervical cytology in the League Against Cancer Lima-Perú, 2010-2014, determining the prevalence of abnormal cytology associated with the socialdemographic characteristics of the patients. Materials and methods. A quantitative, observational, analytical, cross-sectional retrospective study, for a non-experimental design. 206203 cervical cytologies were studied as total population. The data collection instrument was compiled from the clinical history of each patient record. For the analysis, descriptive statistics and analytical statistical tests were used. Results. The mean age was 47 years with a SD \pm 13 years, $1,80 \%$ prevalence of abnormal cytology was found, the highest percentage of this cytology was in the age range of 15 to 24 years with $3.06 \%$, which in turn, it presented the highest percentage of low-grade intraepithelial lesion with $3.04 \%$, and the highest percentage of high-grade intraepithelial lesion was in the age range of 35 to 49 years with $0.34 \%$. It was identified as associated factors, mainly the initiation of sexual relations at an early age ( 13 to 15 years) with a $p=0,001$, the number of sexual partners ( 3 to more) with a $p=0,001$ and the socioeconomic level, at a lower level higher prevalence of abnormal cytology. Conclusions. In conclusion, was identified as factors associated with abnormal cytology, those related to sexuality and socioeconomic status, determining the prevalence of abnormal cytology at $1,80 \%$.
\end{abstract}

Key words: Abnormal cytology; High-grade intraepithelial lesions; Low-grade intraepithelial lesion (Source: MeSH NLM).

\section{INTRODUCCIÓN}

El cáncer es un problema de salud pública a nivel mundial. En el informe de "Prevención de las Enfermedades Crónicas: una Inversión Vital" realizado por la Organización Mundial de la Salud, se calculó que más de 7,5 millones de personas morirían de cáncer en el 2005 y que más del $70 \%$ de dichas defunciones se producirían en países de ingresos bajos y medios. El cáncer de cuello uterino, es uno de los principales en cuanto a mayor incidencia, sobre todo en los países en desarrollo como el nuestro y en general en América

\footnotetext{
Médico Cirujano con especialidad en cirugía general y oncología

Instituto de Investigación de Ciencias Biomédicas (INICIB). Universidad Ricardo Palma. Lima-Perú.

Universidad de San Martín de Porres. Facultad de Medicina Humana. Centro de investigación de epidemiologia clínica y medicina basada en evidencia, Lima-Perú

a Liga contra el cáncer.

b Médico Epidemiólogo.

Centro Nacional de Epidemiología, prevención y control de enfermedades-MINSA.

Universidad Continental, Perú.
}

Citar como: Cossser ME, Loayza MJ, Navarrete PJ. Factores asociados a la citología anormal del cérvix. Liga Contra el Cáncer, Lima Perú, 200-2014. Rev Peru Investig Matern Perinat 2019; 8(1):17-22

DOI: https://doi.org/10.33421/inmp.2019137

Recibido: 07-03-19 Aprobado: 27-03-19 
Latina y el Caribe. En el 2012 se reportó 528000 nuevos casos de cáncer de cuello uterino a nivel mundial, y 266 000 muertes por esta enfermedad, siendo los mayores porcentajes en los países menos desarrollados. En el Perú para para el 2015 se proyectó 5,050 casos nuevos y 1,887 casos de mortalidad ${ }^{1}$.

La Liga Contra el Cáncer, es una asociación privada de carácter benéfico social y sin fin de lucro, su visión es eliminar el cáncer como un problema de salud importante previniéndolos tempranamente. La Liga tiene su sede central en Lima y desarrolla su actividad en todo el Perú gracias a sus 18 Filiales en Provincias. En la sede de Lima, se desarrolla desde hace 65 años el programa de prevención de cáncer, dentro del cual, la detección de cáncer de cuello uterino tiene vital importancia, habiéndose examinado hasta diciembre de 2014, a 1'200,000 mujeres en Lima, con un promedio de los últimos cinco años de 45000 citologías por año, habiéndose detectado más de 2000 casos de cáncer de cérvix².

La Liga Contra el Cáncer recibe pacientes de Lima Metropolitana, provincias y a veces hasta del exterior, hay pacientes que vienen de otras instituciones y también, mayores de 70 años, además de pacientes continuadoras, es decir, que todos los años se realizan el examen citológico. La Liga cuenta con unidades móviles, las cuales acuden a zonas periféricas de la ciudad, y a instituciones, como empresas, municipalidades, ministerios, etc., donde las pacientes al contar con accesos a otros servicios de salud, probablemente ya se han realizado el examen citológico.

La Organización Mundial de la Salud ha publicado en varias ocasiones desde hace más de 20 años, lineamientos generales para el desarrollo de los programas de detección citológica, donde ponen énfasis en dirigir estos exámenes a la población de riesgo para esta enfermedad y así poder bajar la mortalidad ${ }^{3}$. Desde 1943, donde la prueba de citología de cuello uterino toma el nombre de Papanicolaou en homenaje al Dr. George Papanicolaou ${ }^{4}$, este examen causó un gran impacto, en los Estados Unidos entre los años 50 y 70 en donde la incidencia y mortalidad de cáncer de cuello uterino se redujo en más del $70 \%{ }^{5}$. Asimismo, en la Columbia Británica del Canadá, se llegó a disminuir la incidencia y mortalidad de un $78 \%$ a $72 \%{ }^{6}$. Es así, que los programas de detección bien desarrollados han demostrado que es posible disminuir la mortalidad producida por cáncer de cuello uterino ${ }^{3}$, para lo cual se debería seguir las recomendaciones de la Agencia Internacional de Investigación Contra el Cáncer (IARCC), dentro de las cuales está la edad: mujeres entre 35 y 65 años, porque la frecuencia de cáncer cervical en mujeres jóvenes es baja, y además se encuentra lesiones intraepiteliales de bajo grado ${ }^{7}$, también está la frecuencia en la toma de Papanicolaou que deberá ser cada 3 años luego de dos exámenes negativos, lo que resulta ser más efectivo, según la IARCC.
Otro de los factores asociados a esta citología anormal viene hacer los relacionados con la sexualidad: inicio temprano de la vida sexual, el número de parejas sexuales y como un factor de causalidad muy importante, la infección de papiloma virus humano, todo lo cual coincide con las investigaciones revisadas en el presente estudio y la literatura.

Se realizó este estudio en La Liga Contra el Cáncer, sede Lima del 2010-2014 para identificar los factores asociados a la citología anormal del cérvix y a su vez determinar la prevalencia, para finalmente hacer las correcciones y/o recomendaciones para el mejor desarrollo de este programa.

\section{MATERIALES Y METÓDOS}

Se diseñó un estudio cuantitativo, observacional, analítico, retrospectivo, de corte transversal y no experimental. Se tomó como población total a todas las pacientes que tuvieron el examen citológico de Papanicolaou en la Liga Contra el Cáncer sede Lima del 2010-2014, las cuales fueron un total de 216,604 . Se consideró como criterio de exclusión a pacientes con prueba citológica inadecuada, por la cual se excluyó a 10401 pacientes quedando como población de estudio 206203 citologías. El instrumento de recolección de datos fue una ficha confeccionada a partir de la historia clínica de cada paciente.

Las variables evaluadas fueron las relacionadas a la sexualidad, como edad inicio de la primera relación sexual, número de parejas sexuales, también se evaluó el grado de instrucción, ocupación, y se correlacionó con los diagnósticos de lesión intraepitelial de bajo grado (LEI BG) y lesión intraepitelial de alto grado (LEI AG). La variable procedencia se adecuó a la tabla del Asociación Peruana de Investigación de Mercados (APEIM), para establecer los niveles socioeconómicos por grupos de distritos y correlacionarlos con la citología anormal de este estudio. Asimismo, esta misma citología se adecuó al mapa de la pobreza presentada por el INEI en el último semestre del año 2014. Se realizaron análisis descriptivo y se hizo uso de la estadística analítica en donde si la prueba tenia valor de $p>0.05$, se consideraba como estadísticamente significativo. La información de cada historia fue codificada con la finalidad de salvaguardar los nombres y cumplir con los principios éticos en la investigación.

\section{RESULTADOS}

Durante el periodo de estudio se evaluaron 206,203 citologías. Se encontró 3,718 citologías anormales con lo que se tuvo una prevalencia de $1.8 \%$. En las pacientes en las que se realizó por primera vez un Papanicolaou se encontró $1,84 \%$ de prevalencia de citología anormal y en las que ya habían tenido uno o más exámenes $1,66 \%$ de esta citología ( $p=0,014)$. 
Tabla № 1. Relación de Edad y Citología Anormal Liga Contra el Cáncer, Lima-Perú, 2010-2014

\begin{tabular}{|c|c|c|c|c|c|c|}
\hline Edad & $\begin{array}{l}\text { Citología } \\
\text { Anormal }\end{array}$ & $\begin{array}{c}\text { Porcentaje Citología } \\
\text { Anormal }\end{array}$ & $\begin{array}{l}\text { Citología } \\
\text { Normal }\end{array}$ & $\begin{array}{c}\text { Porcentaje Citología } \\
\text { Normal }\end{array}$ & Total & $\begin{array}{l}\text { Porcentaje } \\
\text { Total }\end{array}$ \\
\hline 0-14 años & 0 & $0.00 \%$ & 13 & $0.01 \%$ & 13 & $0.01 \%$ \\
\hline 15-24años & 177 & $3.06 \%$ & 5610 & $2.77 \%$ & 5787 & $2.81 \%$ \\
\hline 25-34 años & 818 & $2.50 \%$ & 31952 & $15.78 \%$ & 32770 & $15.89 \%$ \\
\hline 35-49 años & 1726 & $2.19 \%$ & 77074 & $38.06 \%$ & 78800 & $38.21 \%$ \\
\hline 50-64 años & 832 & $1.24 \%$ & 66469 & $32.83 \%$ & 673001 & $32.64 \%$ \\
\hline 65-79 años & 148 & $0.75 \%$ & 19464 & $9.61 \%$ & 19612 & $9.51 \%$ \\
\hline 80 a más & 17 & $0.89 \%$ & 1903 & 0.94 & 1920 & $0.93 \%$ \\
\hline Total & 3718 & $1.80 \%$ & 202485 & $98.20 \%$ & 206203 & $100 \%$ \\
\hline
\end{tabular}

Fuente: Data Liga Contra el Cáncer

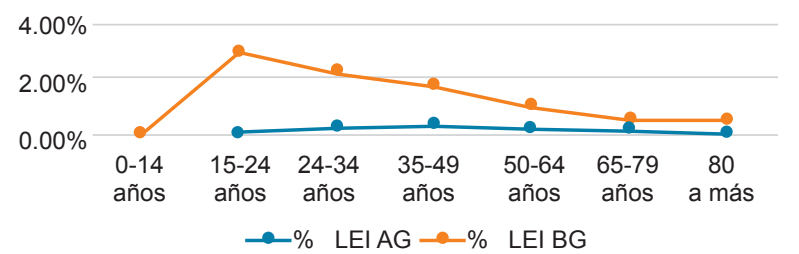

Gráfico No 1. Relación de edad con LEI AG y LEI BG. Liga Contra el Cáncer, Lima-Perú, 2010-2014

En relación a la edad, la citología (PAP) se realizó a pacientes antes de los 14 años hasta más de 80 años de edad, siendo la media 47 años con una desviación estándar de 13 años, encontrándose el mayor porcentaje de citología anormal en los rangos de edades que van de 15 a 24 años, siendo a su vez, esta, lesión intraepitelial de bajo grado $(3,04 \%)$ y en el rango de 35 a 49 años el mayor porcentaje fue la lesión intraepitelial de alto grado (LEI AG). (Ver tabla 1 y gráfico 1). En lo referente a la edad de inicio de las relaciones sexuales, se encontró el mayor porcentaje de citología anormal en el rango de 13 a 15 años con 2,63\%, siendo ésta mayormente para la lesión intraepitelial de bajo grado $(p=0,001)$. Ver gráfico 2.

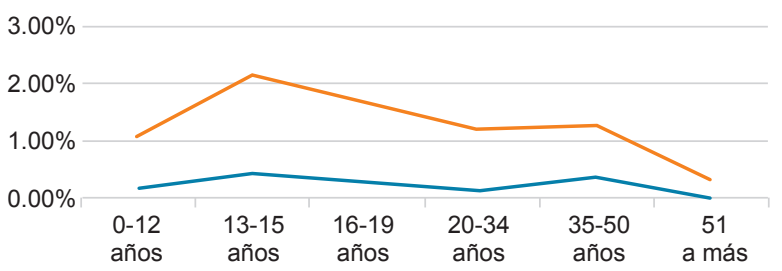

Gráfico № 2. Edad de inicio de relaciones sexuales en relación a citología anormal.

Liga Contra el Cáncer, Lima-Perú, 2010-2014

El estado civil también guardó relación con la citología anormal encontrando $1,31 \%$ de citología anormal para las casadas y $2,16 \%$ para otros estados (conviviente, divorciada, separada, soltera, viuda) con $p=0,001$.

En cuanto al número de parejas sexuales, se encontró una relación directa, a mayor número de parejas, mayor porcentaje de citología anormal, es así: una pareja sexual se encontró $1,35 \%$ de citología anormal, 4 a más parejas sexuales 2,79\% de citología anormal con un $\mathrm{p}=0,001$ (Ver tabla 2).

El grado de instrucción también guardó una relación directa, es así como a mejor grado de instrucción menor porcentaje de citología anormal con un $p=0,027$. En la

Tabla No 2. Citología Anromal en Relación al Número de Parejas Sexuales Liga Contra el Cáncer, Lima- Perú, 20102014.

\begin{tabular}{lcccc}
\hline \multicolumn{1}{c}{ Parejas Sexuales } & $\begin{array}{c}\text { Citología } \\
\text { Anormal }\end{array}$ & Citología Anormal\% & $\begin{array}{c}\text { Citología } \\
\text { Normal }\end{array}$ & Citología Normal \% \\
\hline 1 Pareja sexual & 1116 & $1.35 \%$ & 81503 & $48.06 \%$ \\
2-3 Parejas sexuales & 1640 & $2.13 \%$ & 75396 & $44.46 \%$ \\
4 a más PS & 364 & $2.79 \%$ & 12696 & $7.49 \%$ \\
Total & 3120 & $1.81 \%$ & 169595 & $100 \%$ \\
\hline
\end{tabular}

$\mathrm{p}=0.001$

Fuente: Data Liga Contra el Cáncer 
Tabla N³: Citología Anormal por Niveles Socioeconómicos adecuada a la aTabla de APEIM Liga Contra el Cáncer, Lima- Perú, 2010-2014

\begin{tabular}{|c|c|c|c|c|c|c|c|}
\hline \multirow{2}{*}{ LUGAR DE PROCEDENCIA } & \multirow{2}{*}{ TOTAL } & \multicolumn{5}{|c|}{ NIVELES SOCIOECONÓMICOS } & \multirow{2}{*}{$\begin{array}{l}\text { CITOLOGÍA } \\
\text { ANORMAL \% }\end{array}$} \\
\hline & & NSE A & NSE B & NSE C & NSE D & NSE D & \\
\hline 1 Puente Piedra, Comas, Carabayllo & $100 \%$ & $0.9 \%$ & $12.3 \%$ & $45.4 \%$ & $26.5 \%$ & $14.8 \%$ & $2.18 \%$ \\
\hline $\begin{array}{l}2 \text { Independencia, Los Olivos, San } \\
\text { Martín de Porres }\end{array}$ & $100 \%$ & $2.1 \%$ & $20.5 \%$ & $50.7 \%$ & $21.7 \%$ & $5.0 \%$ & $1.91 \%$ \\
\hline 3 San Juan de Lurigancho & $100 \%$ & $1.9 \%$ & $10.3 \%$ & $41.2 \%$ & $36.6 \%$ & $9.9 \%$ & $1.73 \%$ \\
\hline 4 Cercado, Rímac Breña, La Victoria & $100 \%$ & $1.8 \%$ & $21.6 \%$ & $45.0 \%$ & $25.5 \%$ & $6.1 \%$ & $1.73 \%$ \\
\hline $\begin{array}{l}5 \text { Atye, Chaclacayo, Lurigancho, } \\
\text { Santa Anita, San Luis, El Agustino }\end{array}$ & $100 \%$ & $1.9 \%$ & $11.9 \%$ & $40.2 \%$ & $36.0 \%$ & $10.0 \%$ & $1.88 \%$ \\
\hline $\begin{array}{l}6 \text { Jesús María, Lince, Pueblo Libre, } \\
\text { Magdalena, San Miguel }\end{array}$ & $100 \%$ & $19.9 \%$ & $48.8 \%$ & $22.7 \%$ & $6.5 \%$ & $2.1 \%$ & $1.45 \%$ \\
\hline $\begin{array}{l}7 \text { Miraflores, San Isidro, San Borja, } \\
\text { Surco, La Molina }\end{array}$ & $100 \%$ & $29.0 \%$ & $44.9 \%$ & $18.8 \%$ & $5.0 \%$ & $2.3 \%$ & $1.41 \%$ \\
\hline $\begin{array}{l}8 \text { Surquillo Barranco, Chorrillos, San } \\
\text { Juan de Miraflores }\end{array}$ & $100 \%$ & $4.8 \%$ & $22.1 \%$ & $40.1 \%$ & $24.6 \%$ & $8.5 \%$ & $1.74 \%$ \\
\hline $\begin{array}{l}9 \text { Villa El Salvador, Villa María del } \\
\text { Triunfo, Lurín, Pachacamac }\end{array}$ & $100 \%$ & $0.0 \%$ & $10.0 \%$ & $42.2 \%$ & $36.9 \%$ & $11.0 \%$ & $1.70 \%$ \\
\hline $\begin{array}{l}10 \text { Callao, Bellavista, La Perla, La } \\
\text { Punta, Carmen de la Legua, } \\
\text { Ventanilla }\end{array}$ & $100 \%$ & $1.5 \%$ & $17.2 \%$ & $42.2 \%$ & $25.2 \%$ & $13.9 \%$ & $1.81 \%$ \\
\hline 11 Otros & $100 \%$ & $0.0 \%$ & $8.5 \%$ & $48.9 \%$ & $27.7 \%$ & $14.9 \%$ & 1. $64 \%$ \\
\hline TOTAL & $100 \%$ & $5.20 \%$ & $20.00 \%$ & $40.40 \%$ & $25.70 \%$ & $8.70 \%$ & \\
\hline
\end{tabular}

Fuente: Data Liga Contra el Cáncer y APEIM adaptada por el autor

variable ocupación, su casa, que fue el $57,44 \%$ de nuestra población, se encontró $1,70 \%$ de citología anormal, y en las que referían de ocupación estudiante, el mayor porcentaje de citología anormal con 2,60\%, siendo esta, lesión intraepitelial de bajo grado.

En cuanto al lugar de procedencia, se correlacionó los distritos de Lima, con la tabla de distribución de niveles socioeconómicos por zonas distritales del APEIM para el 2015 de Lima Metropolitana, y a su vez con nuestros resultados de citología anormal por distritos, donde se encontró a menor nivel socioeconómico ( $D, E)$ mayor citología anormal (2,18\%). (Ver tabla 3).

\section{DISCUSIÓN}

El cáncer ya es reconocido como un problema de salud mundial, y dentro esta patología, el de cuello uterino es uno de los principales en cuanto a incidencia, sobre todo en los países en desarrollo como el nuestro, es así como la cifra proyectada para este año (2015) para nuestro país es de 5,050 casos nuevos y la mortalidad de 1,887 casos $^{1}$, por lo tanto, los diversos programas de detección existentes en nuestro país, de distintas instituciones, se han abocado a realizar el screning mediante la prueba del Papanicolaou desde hace muchos años atrás, pero no se ha conseguido bajar la mortalidad. La Liga Contra el Cáncer institución que desde hace 65 años se dedica a la prevención de cáncer, ha realizado 1200000 citologías desde su fundación, y solo en los últimos 5 años 216604 de las cuales se estudió en el presente estudio 206203 y se encontró $1,80 \%$ de prevalencia, porcentaje bajo en comparación a otros estudios como el de la Universidad Industrial de Colombia que en 1,549 estudiantes encontraron una prevalencia de anormalidad de $17,8 \%{ }^{8}$, en el mismo país (Colombia) en 205217 pacientes de un servicio de salud de Medellín encontraron $8,5 \%$ de citología anormal ${ }^{9}$, asimismo en Bolivia en el Hospital Boliviano El Torno encontraron 10,9\% de citología anormal en 886 citologías $^{10}$, sin embargo coincide con un estudio realizado en Lima Perú en las madres del comité del vaso de leche de la Municipalidad de Surquillo donde se encontró $2,2 \%$ de anormalidad citológica en 1,142 mujeres ${ }^{11}$. Como vemos este bajo porcentaje de prevalencia de citología anormal de este estudio puede deberse a un creciente número de pacientes continuadores en relación a las pacientes que por primera vez tuvieron el examen y también de estas últimas muchas vienen de otras instituciones donde ya se habían realizado la citología. Entonces, si han tenido citología anterior una o dos la posibilidad de que esta sea anormal disminuye, de allí las recomendaciones de la OMS, en cuanto a la frecuencia del PAP ${ }^{12}$.

En cuanto a la edad el mayor porcentaje de citología se realizó en el rango de edad de 35 a 64 años $(70,69 \%)$ lo cual coincide con las directivas de la $\mathrm{OMS}^{12}$, sin embargo 
un importante porcentaje se realizó por debajo de los 34 años, y es donde se encontró mayor prevalencia de citología anormal $(3,06 \%)$ coincidente con otros estudios, como el de las estudiantes de Bucaramanga, Colombia ${ }^{8}$, pero a su vez este grupo, presentó lesión intraepitelial de bajo grado tal como refiere la literatura (7). En el grupo de 35 a 49 años es donde se encuentra en mayor porcentaje la prevalencia de citología de lesión intraepitelial de alto grado con un $0,34 \%$, coincidente con la literatura ${ }^{13}, y$ también con un estudio realizado en Medellín Colombia ${ }^{9}$, a mayor edad mayor porcentaje de LEI AG.

En cuanto a la sexualidad, como factor asociado a la citología anormal, cuanto más precoz fue la edad de la primera relación sexual más alto fue el porcentaje de anormalidad, coincidente con el estudio de las estudiantes universitarias de Bucaramanga, Colombia ${ }^{8}$, y también con otro estudio de la Universidad del Cauca Colombia (2007-2008) en el que reporta mayor porcentaje en aquellas que inician su vida sexual antes de los 18 años $^{14}$, asimismo el número de parejas sexuales resultó ser un factor asociado importante es así como, a mayor número de parejas, mayor porcentaje de anormalidad, coincidente con el estudio realizado en las madres del comité del vaso de leche de la Municipalidad de Surquillo, Lima Perú, en donde encontraron un mayor riesgo (1,3 de $\mathrm{OR})$ en aquellas que referían tres o más parejas sexuales ${ }^{11}$, y también con otro estudio realizado en una institución de salud del estado de México (20082010) ${ }^{15}$

En cuanto al estado civil en las casadas el porcentaje de citología anormal $(1,31 \%)$ en relación al de otros estados $(2,16 \%)$ y con un $p=0.001$, es similar con el estudio de las estudiantes universitarias de Bucaramanga, Colombia que reportan una prevalencia de anormalidad de 17,9\% para las solteras y $12,5 \%$ para otros estados ${ }^{8}$, al igual que el estudio en las madres del comité del vaso de leche de la Municipalidad de Surquillo, Lima Perú, donde las solteras presentaban tres veces más riesgos de citología anormal que las casadas ${ }^{11}$.

En cuanto al grado de instrucción como factor asociado en nuestro estudio, cuanto mayor fue el grado, menor fue la citología anormal con un $p=0,027$, no se encontró estudios anteriores específicos contrastables con esta variable.

El factor ocupación su casa que representó más de la mitad de nuestra población $(57,44 \%)$ fue el que menor citología anormal presentó $(1,70 \%)$, y el mayor porcentaje fue para las que reportaron ocupaciones de estudiantes $(2,60 \%)$, contrastable con el estudio de las madres del comité del vaso de leche de la Municipalidad de Surquillo, Lima Perú ${ }^{11}$.

En cuanto a la procedencia, las pacientes de la zonas distritales de menor nivel socioeconómico (D,E) presentaron mayor porcentaje de citología anormal
$(2,18 \%)$ y las pacientes de la zonas de mejor nivel socioeconómico $(A, B)$ menor prevalencia de citología anormal $(1,41 \%)$, esto se puede comparar de alguna manera con el estudio sobre el nivel de pobreza asociado al estadío de gravedad del cáncer ginecológico realizado en el INEN (2000-2004) donde a mayor nivel de pobreza mayor porcentaje de cáncer avanzado de cuello uterino ${ }^{16}$.

En conclusión, los principales factores asociados a la citología anormal en este estudio, fueron la precocidad de la edad de inicio de las relaciones sexuales, el número de parejas sexuales y el nivel socioeconómico: cuando menor era el $\operatorname{NSE}(D, E)$ mayor prevalencia de citología anormal, y se determinó la prevalencia global, en 1,80\% de citología anormal.

Financiamiento: Autofinanciado.

Conflicto de interés: Los autores declaran no tener algún conflicto de intereses.

\section{REFERENCIAS BIBLIOGRÁFICAS}

1. GLOBOCAN 2012. Estimated Cancer Incidence, Mortality and Prevalence Worldwide in 2012, International Agency for Research on Cancer, OMS. http://globocan.iarc.fr/Default. aspx

2. Liga Contra el Cáncer. Informe Memoria. 2014.

3. Miller A. Programas de Detección de Cáncer Cervicouterino: Directrices de Gestión, Organización Mundial de la Salud, Ginebra 1993.

4. Romero N. Reseña Histórica de la Citopatología y los Orígenes del Papanicolaou, Anales de la Facultad de Medicina Universidad Nacional Mayor de San Marcos, 2001, Vol. $2 N^{\circ} 4$, ISSN 1025-5583. http://sisbib.unmsm.edu.pe/ BVRevistas/anales/v62_n4/pdf/a08v62n4.pdf

5. Surveillance, Epidemiology and End Results (SEER) Program: SEER 1973-2000 Public Use Data, Seer Registry 9 database. Availlable at http://surveillance.cancer.gov/ publications/factsheets/New_Malignancies_SEER_Fact_ Sheet.pdf

6. Barriga O. Detección de Cáncer de Cuello Uterino Ginecología y Obstetricia, editor Ludmir, A., Cervantes, R., Castellano, C., Prevención-Diagnostico-Tratamiento, Lima-Perú, Concytec 1996, sección III capítulo 33, tema 90, pag.1128-1129.

7. Ministerio de Salud de Argentina, Guía Programática Abreviada para el Tamizaje de Cáncer de Cuello Uterino para el Programa Nacional de Prevención de Cáncer Cervicouterino, Argentina, enero 2014. http://www.msal. gob.ar/images/stories/bes/graficos/0000000433cnt-Guia Programatica_Abreviada_BAJA.pdf

8. Fajardo-Peña $M$, García-Rueda $A$, Caballero-Badillo $M$, Vargas-Hernández D, Camargo-Figueroa F, Prevalencia y factores asociados al resultado anormal de la citología vaginal en estudiantes universitarias en Bucaramanga, Colombia. Rev. costarric. salud pública. 2013, vol.22, n.2, pp. 127-133. ISSN 1409-1429. http://www.scielo.sa.cr/pdf/rcsp/ v22n2/art07v22n2.pdf

9. Cardona-Arias J, Valencia-Arredondo M. Prevalencia de alteraciones oncológicas en la citología cervicovaginal de pacientes de una institución prestadora de servicios de salud de Medellín, 2010-2012. CES Med. [revista en la Internet]. 
2014 Ene [citado 2015 Sep 06] ; 28( 1 ): 7-20. Disponible en: $\quad$ http://www.scielo.org.co/scielo.php?script=sci arttext\&pid=S0120-87052014000100002\&lng=es

10. Salas I, Tejera G, Ricaño I, Del Prado R. Aspectos epidemiológicos en citologías con resultados anormales en el hospital boliviano "El Torno". (Spanish). Medisan [serial on the Internet]. (2013, Mar), [cited August 31, 2015]; 17(3): 477483. Available from: Academic Search Complete.

11. Bautista F, Vallejos C, Bances G, Galdos O, Santos C. Prevalencia de lesiones premalignas de cuello uterino e infección por papilomavirus humano en madres del Comité del Vaso de Leche de la Municipalidad de Surquillo. 2012;3(1):3-9.

12. Control Integral del Cáncer Cervicouterino: Publicación Occasional: Guía de Prácticas Esenciales, World Health Organization, 2007, http://apps.who.int/iris/ bitstream/10665/43675/1/9789243547008_spa.pdf

13. Castellano C, Santos C, Alvarez M, Mariategui J, Ramire G. Detección de Cáncer de Cuello Uterino Ginecología y Obstetricia, editor Ludmir A, Cervantes R, Castellano C. Prevención-Diagnostico-Tratamiento. Lima-Perú, Concytec 1996, sección III capítulo 28, tema 82, pag.1049-1052.
14. Tafurt-Cardona Y, Acosta-Astaiza CP, Sierra-Torres $\mathrm{CH}$. Prevalencia de citología anormal e inflamación y su asociación con factores de riesgo para neoplasias del cuello uterino en el Cauca, Colombia. Revista de Salud Pública 2012:1453-66. Disponible en: http://www.redalyc.org/articulo. oa?id=42223282005. Fecha de consulta: 6 de septiembre de 2015.

15. Cabrera-Gaytan D, Palacios-Rodriguez R, Guzman-Solorio C. Perfil Sexual de la Mujeres con Citologia Cervical de una unidad de primer nivel, Rev Med Inst Mex Seguro Soc. 2014;52(2):168-75. http://www.medigraphic.com/pdfs/imss/ im-2014/im142n.pdf.

16. Gutierrez C, Alarcon E. Nivel de pobreza asociado al estadio de gravedad del cáncer ginecológico. An Fac med.2008;69(4):239-243. ISSN 1025-5583. http://www. scielo.org.pe/pdf/afm/v69n4/a04v69n4.pdf.

\section{Correspondencia:}

Dirección: Calle Las Estrellas 179, Urb. Alborada, Santiago de Surco.

Teléfono: 924216168 